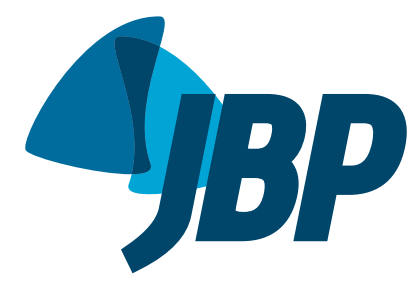
Médicas - LABIMED - Departamento de Radiologia, Pavilhão Pereira Filho, Irmandade Santa Casa de Misericórdia de Porto Alegre, Porto Alegre (RS) Brasil.

2. Programa de Pós-Graduação em Medicina, Pontifícia Universidade Católica do Rio Grande do Sul, Porto Alegre (RS) Brasil.

3. Grupo Fleury, São Paulo (SP) Brasil.

4. Hospital Heliópolis, São Paulo (SP) Brasil.

5. A. C. Camargo Cancer Center, São Paulo (SP) Brasil.

6. Departamento de Radiologia, Universidade Federal do Rio de Janeiro, Rio de Janeiro (RJ) Brasil.

7. Instituto Radiodiagnóstico Rio Preto São José do Rio Preto (SP) Brasil.

a. (iD) http://orcid.org/0000-0002-2536-3874

b. (iD) http://orcid.org/0000-0002-4822-7082

c. (iD http://orcid.org/0000-0001-7851-5125

d. (iD) http://orcid.org/0000-0001-9214-1916

e. (iD http://orcid.org/0000-0002-6948-3982

f. iD http://orcid.org/0000-0002-4985-6374

g. (iD) http://orcid.org/0000-0001-9749-1798

h. (iD) http://orcid.org/0000-0002-4964-4160

i. iD http://orcid.org/0000-0002-9458-5649

j. (iD) http://orcid.org/0000-0001-8797-7380

k. (iD http://orcid.org/0000-0001-8079-6712

I. iD http://orcid.org/0000-0003-1984-4636

Submitted: 25 May 2018.

Accepted: 9 January 2019

Study carried out in the Laboratório de Pesquisas em Imagens Médicas LABIMED - Departamento de Radiologia, Pavilhão Pereira Filho, Irmandade Santa Casa de Misericórdia de Porto Alegre, Porto Alegre (RS) Brasil and under the auspices of the Programa de Pós-Graduação em Medicina, Pontifícia Universidade Católica do Rio Grande do Sul, Porto Alegre (RS) Brasil.

\section{Thoracic calcifications on magnetic resonance imaging: correlations with computed tomography}

Juliana Fischman Zampieri',a, Gabriel Sartori Pacini ${ }^{1, \mathrm{~b}}$, Matheus Zanon ${ }^{1, \mathrm{c}}$, Stephan Philip Leonhardt Altmayer ${ }^{1,2, d}$, Guilherme Watte ${ }^{1,2, e}$, Marcelo Barros ${ }^{1,2, f}$ Evandra Durayski ${ }^{2, g}$, Gustavo de Souza Portes Meirelles ${ }^{3, h}$, Marcos Duarte Guimarães ${ }^{4,5, i}$, Edson Marchiori ${ }^{6, j}$, Arthur Soares Souza Junior ${ }^{7, k}$, Bruno Hochhegger ${ }^{1,2,1}$

\section{ABSTRACT}

Objective: To identify the characteristics of thoracic calcifications on magnetic resonance (MR) imaging, as well as correlations between MR imaging and CT findings. Methods: This was a retrospective study including data on 62 patients undergoing CT scans and MR imaging of the chest at any of seven hospitals in the Brazilian states of Rio Grande do Sul, São Paulo, and Rio de Janeiro between March of 2014 and June of 2016 and presenting with calcifications on CT scans. T1- and T2-weighted MR images (T1- and T2-WIs) were semiquantitatively analyzed, and the lesion-to-muscle signal intensity ratio (LMSIR) was estimated. Differences between neoplastic and non-neoplastic lesions were analyzed. Results: Eighty-four calcified lesions were analyzed. Mean lesion density on CT was $367 \pm 435 \mathrm{HU}$. Median LMSIRs on T1- and T2-WIs were 0.4 (interquartile range [IQR], 0.1-0.7) and 0.2 (IQR, 0.0-0.7), respectively. Most of the lesions were hypointense on T1- and T2-WIs ( $n=52$ [61.9\%] and $n=39$ [46.4\%], respectively). In addition, 19 (22.6\%) were undetectable on T1-WIs (LMSIR $=0$ ) and $36(42.9 \%)$ were undetectable on T2-WIs $($ LMSIR $=0)$. Finally, $15.5 \%$ were hyperintense on T1-WIs and $9.5 \%$ were hyperintense on T2-WIs. Median LMSIR was significantly higher for neoplastic lesions than for non-neoplastic lesions. There was a very weak and statistically insignificant negative correlation between lesion density on $\mathrm{CT}$ and the following variables: signal intensity on T1-WIs, LMSIR on T1-WIs, and signal intensity on T2-WIs $(r=-0.13, p=0.24 ; r=$ $-0.18, p=0.10$; and $r=-0.16, p=0.16$, respectively). Lesion density on CT was weakly but significantly correlated with LMSIR on T2-WIs ( $r=-0.29, p<0.05)$. Conclusions: Thoracic calcifications have variable signal intensity on T1- and T2-weighted MR images, sometimes appearing hyperintense. Lesion density on CT appears to correlate negatively with lesion signal intensity on MR images.

Keywords: Calcification, physiologic; Thorax/diagnostic imaging; Tomography, X-ray computed; Magnetic resonance imaging.

\section{INTRODUCTION}

Thoracic calcifications are associated with various diseases, including calcified granulomas, metabolic disorders, occupational diseases, and lung metastases, as well as benign and malignant tumors. ${ }^{(1-5)} \mathrm{CT}$ is the gold standard method for detecting and characterizing calcifications. ${ }^{(1)}$ There have been few studies of thoracic calcifications on magnetic resonance (MR) imaging, most reports of calcifications on MR imaging being related to intracranial lesions. ${ }^{(6-10)}$

Calcifications generate nonspecific signal intensities on conventional T1- and T2-weighted images (WIs) and gradient-echo images. ${ }^{(7,8,11)}$ Because calcium salts do not contain mobile protons, they have no signal on MR images, and densely calcified lesions have been classically described as having low signal intensity on T1- and T2-WIs. ${ }^{(6-8,11)}$ However, studies have reported hyperintense, hypointense, and isointense signals on both T1- and T2-WIs, signal intensity depending on the specific composition of aggregates of calcium salts and on particle size. ${ }^{(7,8,11,12)}$

Although X-rays and CT scans have been extensively used for thoracic evaluation, MR imaging of the chest is an emerging modality. ${ }^{(13-17)}$ It combines functional and

Correspondence to:

Bruno Hochhegger. Laboratório de Pesquisas em Imagens Médicas - LABIMED - Departamento de Radiologia, Pavilhão Pereira Filho, Irmandade Santa Casa de Misericórdia de Porto Alegre, Avenida Independência, 75, CEP 90020160, Porto Alegre, RS, Brasil.

Tel.: 5551 3214-8300. E-mail: brunoho@ufcspa.edu.br

Financial support: None. 
morphological imaging and is therefore an alternative for patients in whom exposure to ionizing radiation is a concern, including children, pregnant women, and patients undergoing repeat examinations over a long period of time. ${ }^{(13,14)}$ The primary objective of the present study was to identify the characteristics of thoracic calcifications on MR imaging, as well as correlations between MR imaging and $C T$ findings. A secondary objective was to compare calcifications associated with cancer and those not associated with cancer in terms of their MR imaging features.

\section{METHODS}

\section{Study participants}

This was a retrospective study including data on all patients undergoing CT scans and MR imaging of the chest at any of seven hospitals in the Brazilian states of Rio Grande do Sul, São Paulo, and Rio de Janeiro between March of 2014 and June of 2016. The study was approved by the local research ethics committee (Protocol no. 22758413.8.0000.5335), and the requirement for informed consent was waived.

The inclusion criteria were as follows: thoracic calcifications larger than $0.3 \mathrm{~cm}$ on CT scans of the chest and diagnostic-quality chest MR imaging. All of the patients in our study sample had participated in previous studies comparing CT and MR imaging in the assessment of lung nodules and pulmonary vessels, as well as for lung cancer staging. MR imaging and CT scans of the chest were performed in the same week. All patient medical records were reviewed, and lung lesions were classified as neoplastic or non-neoplastic.

\section{MR imaging and CT protocols}

All CT examinations were performed with a 64-row multidetector CT scanner (LightSpeed VCT; GE Healthcare, Chicago, IL, USA), the following parameters being used: $120 \mathrm{kVp}$; $250 \mathrm{~mA}$; rotation time, $0.8 \mathrm{~s}$; and pitch, 1.375 . Volumetric inspiratory CT scans were acquired with $1-\mathrm{mm}$ collimation at $1-\mathrm{mm}$ increments and a soft reconstruction algorithm. All CT scans were obtained with mediastinal window settings (width, 350-450 HU; level, 20-40 HU) and lung parenchymal window settings (width, 1,200-1,600 HU; level, -500 $\mathrm{HU}$ to $-700 \mathrm{HU})$, reconstructions being performed in the axial and coronal planes.

MR imaging was performed with a 1.5-T scanner (Magnetom Aera; Siemens Healthineers, Erlangen, Germany). A dedicated 12-element integrated matrix coil system covering the entire thorax was used for signal reception. (18) The system consists of two flexible phased-array coils (one anterior coil and one posterior coil), each containing a set of six receiver elements. ${ }^{(18)}$ A half-Fourier acquisition single-shot turbo spin-echo (HASTE) sequence was used, and the field of view was adapted to each patient. The HASTE sequence was performed with respiratory gating based on diaphragm navigator. Sequence parameters were as follows: repetition time (TR)/echo time (TE)/ flip angle, infinite $/ 92 \mathrm{~ms} / 150^{\circ}$; parallel acquisition factor, 2; slice thickness, $5 \mathrm{~mm}$; distance factor, $20 \%$; transverse and coronal orientations (matrix sizes, 380 $\times 256$ and $400 \times 320$, respectively); and acquisition time, approximately $90 \mathrm{~s}$. A volumetric interpolated breath-hold examination sequence was used for fast T1-weighted imaging. Sequence parameters were as follows: TR/TE, $5.12 \mathrm{~ms} / 2.51 \mathrm{~ms}$; flip angle, $10^{\circ}$; partition thickness, $5 \mathrm{~mm}$ with no interslice gap; and matrix size, $256 \times 116$ (a three-dimensional breath-hold sequence being used). A T2-weighted fat-saturated periodically rotated overlapping parallel lines with enhanced reconstruction sequence (BLADE; Siemens Healthineers) was also used, sequence parameters being as follows: TR/TE, 4,670 ms/113 $\mathrm{ms}$; and partition thickness, $5 \mathrm{~mm}$ with no interslice gap. Diffusion-weighted imaging was performed with the use of a single-shot echo-planar technique, a slice thickness of $6 \mathrm{~mm}$, spectral attenuated inversion recovery, and respiratory-triggered scanning being used. Sequence parameters were as follows: TR/ TE/flip angle, 3,000-4,500 ms/65 ms/90; diffusion gradient encoding in three orthogonal directions; $b$ $=50,400$, and $800 \mathrm{~s} / \mathrm{mm}^{2}$; field of view, $350 \mathrm{~mm}$; and matrix size, $128 \times 128$. The mean overall time spent in the MR imaging room was approximately 15 min, and no sedation was required.

\section{Image analysis}

The CT scans and MR images were independently reviewed by two chest radiologists who had more than 7 years of experience and who were blinded to patient clinical information. Subsequently, the two radiologists together reviewed the scans and images in order to make a final consensus decision. The criteria for CT and $M R$ imaging findings were those defined in the Fleischner Society Glossary of Terms. ${ }^{(19)}$

During CT analysis, the chest radiologists identified areas of calcification in a mediastinal window, measuring mean density within a region of interest (ROI) that included at least $90 \%$ of the calcification identified on CT. Subsequently, thoracic calcifications were classified as diffuse (diffuse or patchy areas of bone-like calcification), punctate (micronodular areas of bone-like calcification), or laminar (linear areas of bone-like calcification). All scans were reviewed on a dedicated workstation (Advantage Workstation 4.2; GE Healthcare), a picture archiving and communication system being used.

MR images were semiquantitatively analyzed by the aforementioned radiologists, who manually defined three-dimensional ROIs by compounding two-dimensional lesion boundaries drawn on consecutive sections, the calcifications seen on CT being used as reference. In addition, an ROI was drawn in the paraspinal muscle at the same level on axial T1- and T2-WIs (mean area, 50-80 $\mathrm{mm}^{2}$, i.e., 14-30 pixels), and the lesion-to-muscle signal intensity ratio (LMSIR) was estimated. An LMSIR of 
$<1$ characterized a hypointense lesion, an LMSIR = 1 characterized an isointense lesion, an LMSIR > 1 characterized a hyperintense lesion, and an LMSIR $=0$ characterized a lesion that was undetectable by MR imaging (null effect).

\section{Statistical analysis}

All statistical analyses were performed with the Statistical Package for the Social Sciences, version 11.0 (SPSS Inc., Chicago, IL, USA). Continuous variables were expressed as means \pm standard deviations or medians and interquartile ranges (IQRs), whereas categorical variables were expressed as absolute numbers and proportions. Continuous variables with normal distribution were compared by the Student's t-test for independent samples, whereas continuous variables with non-normal distribution were compared by the Mann-Whitney $U$ test. Categorical variables were compared by Fisher's exact test. The level of significance was set at $p<0.05$.

Pearson's correlation coefficients were used in order to assess correlations between continuous variables, coefficients of $0.00-0.20$ indicating a very weak correlation, coefficients $\geq 0.20-0.40$ indicating a weak correlation, coefficients $\geq 0.40-0.70$ indicating a moderate correlation, coefficients $\geq 0.70-0.90$ indicating a strong correlation, and coefficients $\geq$ 0.90 indicating a very strong correlation. ${ }^{(20)}$

\section{RESULTS}

The study sample consisted of 62 patients ( 84 calcified lesions). Of those 62 patients, 37 (59.7\%) were female. Mean ROI size was $22 \mathrm{~mm}^{2}$ (8-49 $\left.\mathrm{mm}^{2}\right)$. Of the 84 lesions, 46 (54.8\%) were solitary. Of the 62 patients in the study sample, 36 had participated in a study comparing CT and MR imaging in the assessment of lung nodules, 18 had participated in a study comparing CT and MR imaging in the assessment of pulmonary vessels, and 8 had participated in a study comparing $\mathrm{CT}$ and MR imaging for lung cancer staging.

Mean lesion density on CT was $367 \pm 435 \mathrm{HU}$. Of the 84 lesions analyzed, $56(66.7 \%)$ were pulmonary lesions, 5 (5.9\%) were pleural lesions, and 23 (27.4\%) were mediastinal lesions. In addition, $65(77.4 \%)$ were diffuse, 15 (17.8\%) were laminar, and 4 (4.8\%) were punctate. Median LMSIRs on T1- and T2-WIs were 0.4 (IQR, 0.1-0.7) and 0.2 (IQR, 0.0-0.7), respectively.

Most of the lesions were hypointense on T1- and T2-WIs ( $n=52$ [61.9\%] and $n=39$ [46.4\%], respectively). In addition, 19 (22.6\%) were undetectable on T1-WIs (LMSIR = 0) and $36(42.9 \%)$ were undetectable on T2-WIs (LMSIR $=0$ ). Finally, $15.5 \%$ were hyperintense on T1-WIs and $9.5 \%$ were hyperintense on T2-WIs (Figure 1).

As can be seen in Table 1, there was a significant difference between neoplastic and non-neoplastic lesions regarding minimum lesion diameter and maximum lesion diameter $(p<0.05)$. Although neoplastic and non-neoplastic lesions were hypointense on T1- and T2-WIs, median LMSIR was significantly higher for the former than for the latter (Table 1). Although all of the neoplastic lesions were detectable by MR imaging (Figures 1 and 2), 25\% and $47.4 \%$ of the non-neoplastic lesions were undetectable on T1- and T2-WIs, respectively (Table 1 ).

Table 2 shows LMSIRs on T1- and T2-WIs, by calcification type. Most of the diffuse calcifications were either hypointense or undetectable on T1- and T2-WIs. Most of the laminar calcifications were hypointense, and all showed signal intensity on T1- and T2-WIs.

There was a very weak and statistically insignificant negative correlation between lesion density on CT and the following variables: signal intensity on T1-WIs, LMSIR on T1-WIs, and signal intensity on T2-WIs ( $r$ $=-0.13, \mathrm{p}=0.24 ; \mathrm{r}=-0.18, \mathrm{p}=0.10 ;$ and $\mathrm{r}=$ $-0.16, p=0.16$, respectively). In addition, lesion density on CT was weakly but significantly correlated with LMSIR on T2-WIs $(r=-0.29, p<0.05)$.

\section{DISCUSSION}

The present study showed that thoracic calcifications have variable signal intensity on T1- and T2-weighted MR images. The chemical composition of calcifications includes crystalline calcium phosphate and hydroxyapatite, as well as a small quantity of copper, manganese, zinc, magnesium, and iron. ${ }^{(8)}$ The fact that the concentrations vary in different physiological
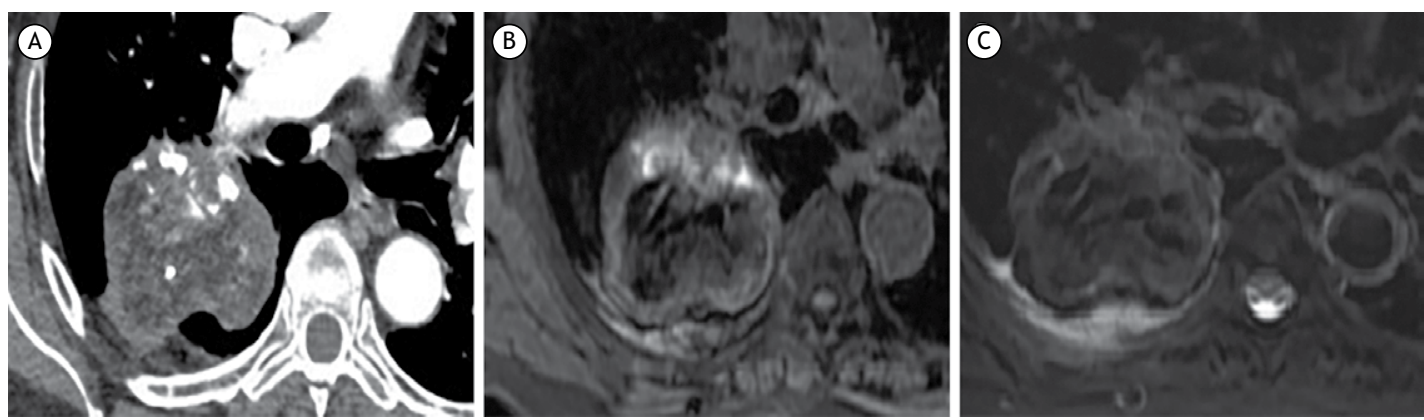

Figure 1. In A, axial CT scan of the chest (mediastinal window) showing calcification within a large lesion in the right lower lobe. In B and C, magnetic resonance imaging of the calcification seen on CT. Note that the lesion is hyperintense on T1- and T2-weighted images ( $\mathrm{B}$ and $\mathrm{C}$, respectively). 
Table 1. Magnetic resonance imaging features of neoplastic and non-neoplastic lung lesions. ${ }^{\mathrm{a}}$

\begin{tabular}{|c|c|c|c|}
\hline Variable & Neoplastic lesions ( $\mathrm{n}=8$ ) & Non-neoplastic lesions $(n=76)$ & p* \\
\hline \multicolumn{4}{|l|}{ Lesion diameter, mm } \\
\hline Minimum diameter & $6.49 \pm 7.72$ & $1.85 \pm 2.75$ & $<0.05$ \\
\hline Maximum diameter & $11.35 \pm 13.20$ & $3.67 \pm 4.96$ & $<0.05$ \\
\hline LMSIR on T1-WIs & $0.9[0.7-1.1]$ & $0.4[0.1-0.6]$ & $<0.05$ \\
\hline Undetectable lesion $(\text { LMSIR }=0)^{c}$ & - & $19(25)$ & \\
\hline Hypointense lesion $(\text { LMSIR }<1)^{c}$ & $5(62.5)$ & $47(61.8)$ & - \\
\hline Isointense lesion $(\mathrm{LMSIR}=1)^{\mathrm{c}}$ & - & $4(5.3)$ & - \\
\hline Hyperintense lesion $(L M S I R>1)^{c}$ & $3(37.5)$ & $6(7.9)$ & - \\
\hline LMSIR on T2-WIs & $0.9[0.4-1.3]$ & $0.1[0.0-0.6]$ & $<0.05$ \\
\hline Undetectable lesion $(\text { LMSIR }=0)^{c}$ & - & $36(47.4)$ & \\
\hline Hypointense lesion $(\text { LMSIR }<1)^{c}$ & $4(50.0)$ & $34(44.7)$ & - \\
\hline Isointense lesion $(\text { LMSIR }=1)^{c}$ & $1(12.5)$ & $2(2.6)$ & - \\
\hline Hyperintense lesion $(\text { LMSIR }>1)^{c}$ & $3(37.5)$ & $4(5.3)$ & - \\
\hline
\end{tabular}

LMSIR: lesion-to-muscle signal intensity ratio; T1-WIs: T1-weighted images; and T2-WIs: T2-weighted images. a Data expressed as mean $\pm \mathrm{SD}$, except where otherwise indicated. 'bata expressed as median [interquartile range]. 'Data expressed as $\mathrm{n}(\%)$. *Mann-Whitney $\mathrm{U}$ test.
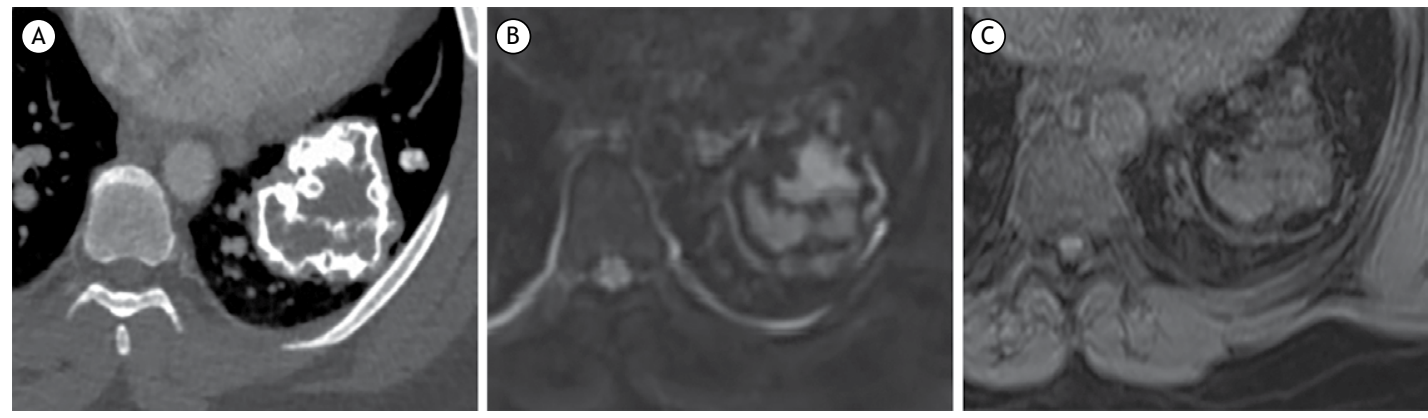

Figure 2. In $A$, axial CT scan of the chest (mediastinal window) showing a large lesion with associated calcifications in the left lower lobe. In B, axial T2-weighted image showing that the calcifications have a markedly lower signal intensity than that of skeletal muscle. In C, axial T1-weighted image showing hypointense calcifications within the lesion.

Table 2. Lesion-to-muscle signal intensity ratios on T1- and T2-weighted images, by calcification type. ${ }^{a}$

\begin{tabular}{|c|c|c|c|}
\hline Variable & $\begin{array}{c}\text { Diffuse } \\
\text { calcifications } \\
(\mathrm{n}=65)\end{array}$ & $\begin{array}{l}\text { Punctate } \\
\text { calcifications } \\
\text { (n = 4) }\end{array}$ & $\begin{array}{l}\text { Laminar } \\
\text { calcifications } \\
(n=15)\end{array}$ \\
\hline LMSIR on T1-WIs & $0.3[0.0-0.5]$ & $0.7[0.5-0.9]$ & $0.6[0.5-0.9]$ \\
\hline Undetectable lesion (LMSIR $=0)^{\mathrm{b}}$ & $18(27.7)$ & $1(25.0)$ & - \\
\hline Hypointense lesion $(\text { LMSIR }<1)^{\mathrm{b}}$ & $39(60.0)$ & $2(50.0)$ & $11(73.3)$ \\
\hline Isointense lesion $(\text { LMSIR }=1)^{b}$ & - & - & - \\
\hline Hyperintense lesion (LMSIR > 1) & $8(12.3)$ & $1(25.0)$ & $4(26.7)$ \\
\hline LMSIR on T2-WIs & $0.0[0.0-0.6]$ & $0.6[0.1-1.3]$ & $0.7[0.4-0.9]$ \\
\hline Undetectable lesion (LMSIR $=0)^{\mathrm{b}}$ & $35(53.8)$ & $1(25.0)$ & - \\
\hline Hypointense lesion $(\text { LMSIR }<1)^{\mathrm{b}}$ & $26(40.0)$ & $1(25.0)$ & $12(80.0)$ \\
\hline Isointense lesion $(\text { LMSIR }=1)^{b}$ & - & - & $1(6.7)$ \\
\hline Hyperintense lesion $(\text { LMSIR }>1)^{b}$ & $4(6.2)$ & $2(50.0)$ & $2(13.3)$ \\
\hline
\end{tabular}

LMSIR: lesion-to-muscle signal intensity ratio; T1-WIs: T1-weighted images; and T2-WIs: T2-weighted images. a Data expressed as median [interquartile range], except where otherwise indicated. ${ }^{\mathrm{b} D a t a}$ expressed as $\mathrm{n}(\%)$.

and pathological calcifications might explain their heterogeneous appearance on MR images. ${ }^{(8)}$ It has been suggested that this variation in appearance is due to a surface-relaxation mechanism, which reduces T1 and $\mathrm{T} 2$ relaxation times. ${ }^{(6)}$ It has been demonstrated that materials that have the same size and chemical composition can have markedly different effects on relaxation depending on the degree of irregularity of the surface. ${ }^{(6,8)}$ The inherent properties of calcium can cause hyperintensity on T1-WIs. The T1 effect predominates in cases in which the surface is very irregular and the surface area is very large-as is the case with calcium crystals-increasing MR signal intensity. ${ }^{(6)}$ This might explain why the proportion of hyperintense lesions was higher on T1-WIs than on T2-WIs in the present study. 
As the calcium concentration increases above $30-40 \%$, proton density decreases, thus resulting in a progressive decrease in signal intensity. ${ }^{(6)}$ The fact that calcified lesions appear hypointense on MR images has been attributed to decreased proton density. ${ }^{(6-10)}$ In the present study, a negative but statistically insignificant correlation was found between lesion density on $\mathrm{CT}$ and lesion signal intensity on MR images, corroborating the finding that lesions with increased calcium concentration tend to have decreased signal intensity on MR images. This could also explain why median LMSIRs on T1- and T2-WIs were lower for diffuse calcifications than for punctate and laminar calcifications.

Previous studies have described neoplastic calcifications appearing hyperintense on MR images. $(7,8,10,21)$ In the present study, neoplastic calcifications had a variable appearance on MR images. However, median LMSIR was significantly higher for hyperintense lesions than for hypointense lesions ( $37.5 \%$ vs. $7.9 \%$ on T1-WIs and $37.5 \%$ vs. $5.3 \%$ on T2-WIs). This might be due to low calcium concentrations changing the surface effects of diamagnetic particles on the MR signal and resulting in $\mathrm{T} 1$ shortening of water protons.

Our study has limitations, some of which are inherent to its retrospective nature. Larger, prospective studies are needed in order to confirm the findings of our subgroup analysis. In addition, future studies should include imaging sequences other than conventional spin-echo T1- and T2-weighted sequences.
Technical challenges to successful MR imaging of the lung include low tissue density (resulting in decreased signal intensity) and magnetic susceptibility differences between tissue and air. ${ }^{(22)}$ Some studies have used gradient-echo MR imaging and quantitative susceptibility mapping in order to characterize calcified brain lesions. (7) Susceptibility-weighted imaging (SWI) is useful for differentiating between intracranial calcifications and hemorrhages, which can have similar attenuation on CT scans. ${ }^{(7)}$ To our knowledge, however, there have been no studies examining the use of SWI in MR imaging of the chest.

Another limitation of our study is the use of a mean signal intensity within a predefined ROI. Signal intensity has been reported to vary throughout a calcified lesion (e.g., a hyperintense periphery and decreased intensity toward the center). ${ }^{(6)}$ However, we believe that this has little impact on clinical practice.

In conclusion, thoracic calcifications (particularly neoplastic calcifications) have variable signal intensity on T1- and T2-weighted MR images, and lesion density on CT appears to correlate negatively with lesion signal intensity on MR images. Radiologists should be aware of these findings when interpreting chest MR images.

\section{ACKNOWLEDGMENTS}

We would like to thank Professor Hans Ulrich Kauczor for his suggestions.

\section{REFERENCES}

1. Bendayan D, Barziv $Y$, Kramer MR. Pulmonary calcifications: a review. Respir Med. 2000;94(3):190-3. https://doi.org/10.1053/ rmed.1999.0716

2. Winer-Muram HT. The solitary pulmonary nodule. Radiology. 2006;239(1):34-49. https://doi.org/10.1148/radiol.2391050343

3. Khan AN, Al-Jahdali HH, Allen CM, Irion KL, Al-Ghanem S, Koteyar SS. The calcified lung nodule: What does it mean? Ann Thorac Med. 2010;5(2):67-79. https://doi.org/10.4103/1817-1737.62469

4. Ceylan N, Bayraktaroglu S, Savas R, Alper H. CT findings of high-attenuation pulmonary abnormalities. Insights Imaging 2010;1 (4):287-292. https://doi.org/10.1007/s13244-010-0039-2

5. Taguchi $Y$, Fuyuno G, Shioya $S$, Yanagimachi N, Katoh $H$, Matsuyama $S$, et al. MR appearance of pulmonary metastatic calcification. J Comput Assist Tomogr. 1996;20(1):38-41. https:// doi.org/10.1097/00004728-199601000-00008

6. Henkelman RM, Watts JF, Kucharczyk W. High signal intensity in RM images of calcified brain tissue. Radiology. 1991;179(1):199206. https://doi.org/10.1148/radiology.179.1.1848714

7. Chen W, Zhu W, Kovanlikaya I, Kovanlikaya A, Liu T, Wang S, et al. Intracranial calcifications and hemorrhages: characterization with quantitative susceptibility mapping. Radiology. 2014;270(2):496505. https://doi.org/10.1148/radiol.13122640

8. Wu Z, Mittal S, Kish K, Yu Y, Hu J, Haacke EM. Identification of calcification with MRI using susceptibility-weighted imaging: a case study. J Magn Reson Imaging. 2009;29(1):177-82. https://doi. org/10.1002/jmri.21617

9. Iglesia MM, Cortés GL, Icaran DG, Cortes IL, Oleaga L, Isusi M. Calcificaciones intracraneales: Imagen por RM. Radiología. 2006;48(1):19-26. https://doi.org/10.1016/S0033-8338(06)73125-3

10. Hochhegger B, Marchiori E, Soares Souza A Jr, Soares Souza
L, Palermo L. MRI and CT findings of metastatic pulmonary calcification. $\mathrm{Br} J$ Radiol. 2012;85(1011):e69-72. https://doi. org/10.1259/bjr/53649455

11. Gamsu G, De Geer G, Cann C, Muller N, Brito A. A preliminary study of $\mathrm{MRI}$ quantification of simulated calcified pulmonary nodules. Invest Radiol. 1987;22(11):853-8. https://doi.org/10.1097/00004424198711000-00001

12. Yi CA, Jeon TY, Lee KS, Lee JH, Seo JB, Kim YK, et al. 3-T MRI usefulness for evaluating primary lung cancer and small nodules in lobes not containing primary tumors. AJR Am J Roentgenol. 2007;189(2):386-92. https://doi.org/10.2214/AJR.07.2082

13. Wild JM, Marshall H, Bock M, Schad LR, Jakob PM, Puderbach $M$, et al. $M R I$ of the lung (1/3): methods. Insights Imaging 2012;3(4):345-53. https://doi.org/10.1007/s13244-012-0176-x

14. Biederer J, Beer M, Hirsch W, Wild J, Fabel M, Puderbach $M$, et al. MRI of the lung (2/3). Why ...when ... how? Insights Imaging 2012;3(4):355-71. https://doi.org/10.1007/s13244-011-0146-8

15. Barreto MM, Rafful PP, Rodrigues RS, Zanetti G, Hochhegger B, Souza AS Jr, et al. Correlation between computed tomographic and magnetic resonance imaging findings of parenchymal lung diseases. Eur J Radiol. 2013;82(9):e492-501. https://doi. org/10.1016/j.ejrad.2013.04.037

16. Pasin L, Zanon M, Moreira J, Moreira AL, Watte G, Marchiori E, et al. Magnetic Resonance Imaging of Pulmonary Embolism: Diagnostic Accuracy of Unenhanced MR and Influence in Mortality Rates. Lung. 2017;195(2):193-199. https://doi.org/10.1007/s00408017-9975-7

17. Weinreb JC. Which study when? Is Gadolinium-enhanced MR imaging safer than iodine-enhanced CT? Radiology. 2008;249(1):38. https://doi.org/10.1148/radiol.2491080075 
18. Maglione M, Montella S, Mollica C, Carnovale V, lacotucci $P$, De Gregorio $F$, et al. Lung structure and function similarities between primary ciliary dyskinesia and mild cystic fibrosis: a pilot study. Ital J Pediatr. 2017;43(1):34. https://doi.org/10.1186/s13052-017-0351-2

19. Hansell DM, Bankier AA, MacMahon H, McLoud TC, Müller NL, Remy J. Fleischner Society: glossary of terms for thoracic imaging. Radiology. 2008;246(3):697-722. https://doi.org/10.1148/ radiol.2462070712

20. Mukaka MM. Statistics corner: A guide to appropriate use of correlation coefficient in medical research. Malawi Med $J$
2012;24(3):69-71.

21. Henz Concatto N, Watte G, Marchiori E, Irion K, Felicetti JC Camargo $\mathrm{JJ}$, et al. Magnetic resonance imaging of pulmonary nodules: accuracy in a granulomatous disease-endemic region. 2015. Eur Radiol. 2016;26(9):2915-20. https://doi.org/10.1007/ s00330-015-4125-1

22. Hochhegger B, de Souza WV, Marchiori E, Irion KL, Souza AS Jr, Elias Junior J, et al. Chest magnetic resonance imaging: a protocol suggestion. Radiol Bras. 2015;48(6):373-80. https://doi. org/10.1590/0100-3984.2014.0017 